\title{
Improvement of the Output Mode Purity of a Complex-Cavity Resonator for a Frequency-Tunable Sub-THz Gyrotron
}

\author{
Dietmar Wagner ，Member, IEEE, and Manfred Thumm ，Life Fellow, IEEE
}

\begin{abstract}
One way to improve the mode selection in terahertz gyrotrons is the use of stepped cavity resonators that satisfy the resonance condition for two modes with the same azimuthal but different radial indices. Such sectioned resonators, or complex cavities, were analyzed in detail by several authors, recently and in the past. One common problem for such designs is the excitation of unwanted modes by both the stepped cavity structure and the cavity input and output tapers. Mode conversion becomes especially a severe problem for higher-order resonant modes in highly oversized resonators. In this article, we introduce ways to minimize the unwanted mode conversion in both the radial step of the cavity as well as in the tapered sections of the cavity and the up-taper to the collector and output window. Frequency tuning between 391.5 and $393.5 \mathrm{GHz}$ has been achieved by operation at different axial mode numbers of the mode pair $\mathrm{TE}_{8,4} / \mathrm{TE}_{8,5}$. Due to their intrinsic higher output mode purity, the so-called antiphase modes of the complex cavity have been chosen for this output mode purity study.
\end{abstract}

Index Terms-Complex cavity resonator, frequency tuning, gyrotron, output mode purity.

\section{INTRODUCTION}

C OMPLEX stepped cavities can improve the mode selectivity in very high-frequency low-power gyrotrons [1]-[3]. Such types of mode converting cavities have been investigated in much detail in the past [4]-[12]. It is well known that in the case of sub-terahertz $(\mathrm{THz})$ gyrotrons, the mechanical precision of the relative ratio of the two cavity radii must be in the order of $10^{-4}$, which is approx. $1 / Q$, where $Q$ is in the order of the quality factors of the two cavity sections. However, even if this mechanical accuracy is achieved, there still remains the question of spurious mode excitation by the cavity step and the output taper of the

Manuscript received May 21, 2021; revised July 19, 2021 and August 11, 2021; accepted August 15, 2021. Date of publication August 27, 2021; date of current version September 22, 2021. The review of this article was arranged by Editor J. Feng. (Corresponding author: Dietmar Wagner.)

Dietmar Wagner is with the Tokamak Scenario Development, Max-Planck-Institute for Plasma Physics, 85748 Garching, Germany (e-mail: dietmar.wagner@ipp.mpg.de).

Manfred Thumm is with the Institute for Pulsed Power and Microwave Technology, Karlsruhe Institute of Technology, 76131 Karlsruhe, Germany (e-mail: manfred.thumm@kit.edu).

Color versions of one or more figures in this article are available at https://doi.org/10.1109/TED.2021.3105955.

Digital Object Identifier 10.1109/TED.2021.3105955 second section. This article deals with this problem and proposes methods to improve the output mode purity of a frequency-tunable sub- $\mathrm{THz}$ gyrotron with complex cavity. Frequency tuning over $2 \mathrm{GHz}$ is achieved by operation on different axial cavity modes $(q=1-5)$. In all cases, an output mode purity $\geq 92.5 \%$ of the gyrotron could be demonstrated. When the frequencies of the partial modes in the two cavity sections are close, their superposition causes formation of normal modes (in-phase and antiphase mode) [3]. For the in-phase mode, the phase is nearly constant at the junction of the two sections, while for the antiphase mode, it exhibits an abrupt sharp change of $\pi$. The features of these two normal modes were studied very carefully in [3], where it was shown that the diffractive quality factors $Q_{\text {dif }}$ for the antiphase modes are much larger than those for the in-phase ones. With the increase of the axial index $q, Q_{\text {dif }}$ of the antiphase modes decreases approximately as $q^{-2}$, whereas for the in-phase modes $Q_{\text {dif }}$ decreases more slowly. The Ohmic quality factors $Q_{\text {ohm }}$ of both normal modes are nearly the same and they slightly increase with $q$. The $\mathrm{TE}_{8,4}$ mode slightly penetrates into the second cavity section where it propagates far from cutoff as a traveling forward wave. For the in-phase mode, this penetration is much stronger than for the antiphase one, and the amplitude of the $\mathrm{TE}_{8,4}$ mode is nearly the same or even larger as the amplitude of the outgoing $\mathrm{TE}_{8,5}$ wave. This is the reason for the lower $Q_{\text {dif }}$ of the in-phase mode. Since we, here in this article, deal with output mode purity, we selected the antiphase mode for the further considerations.

\section{Multimode Cavity Analysis}

As an example, we took the cavity dimensions given in [3] for a complex gyrotron cavity with resonant coupling of the $\mathrm{TE}_{8,4, q} / \mathrm{TE}_{8,5, q}$ modes. The wall radii of the resonant section of the cavity are $R_{1}=2.588 \mathrm{~mm}$ and $R_{2}=2.9974 \mathrm{~mm}$, respectively. The length of both resonant sections $L_{1}$ and $L_{2}$ is $17.5 \mathrm{~mm}$ and the down-tapering and up-tapering angles are $\theta_{1}=2^{\circ}$ and $\theta_{2}=3^{\circ}$, respectively. The cavity geometry is plotted in Fig. 1. The cavity was simulated using a resonator code based on the scattering matrix formalism [13] taking into account all propagating and additional evanescent modes. In particular, for the following calculations, we took into account up to $12 \mathrm{TE}$ and $12 \mathrm{TM}$ modes. The results of these 


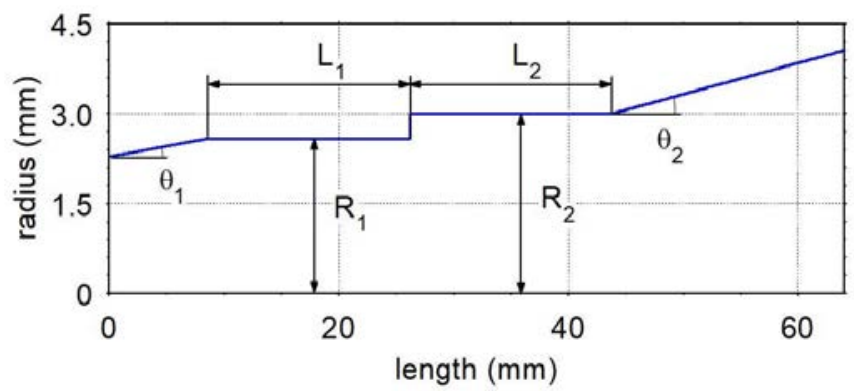

Fig. 1. Radial contour of the complex $\mathrm{TE}_{8,4, q} / \mathrm{TE}_{8,5, q}$ mode gyrotron cavity from [3].

TABLE I

Comparison of the Calculated Resonance Frequencies and DifFRACTIVE QUALITY FACTORS ( $f_{\text {res }}^{*}$ AND $Q_{\text {dif }}^{*}$ ) OF THE FIRST FIVE ANTIPHASE AXIAL MOdEs From [3] WITH THE CORRESPONDING Values ( $f_{\text {res }}$ AND $Q_{\text {dif }}$ ) Calculated HeRE Using the MULTIMODE SCATTERING MATRIX FORMALISM. THE Obtained Output Mode Purities Are Given IN THE LAST COLUMN

\begin{tabular}{|c|c|c|c|c|c|}
\hline $\mathrm{q}$ & $f_{\text {res }}{ }^{*}(\mathrm{GHz})$ & $Q_{\text {dif }}{ }^{*}$ & $f_{\text {res }}(\mathrm{GHz})$ & $Q_{\text {dif }}$ & $\mathrm{TE}_{8,5}(\%)$ \\
\hline 1 & 391.462 & 45002 & 391.474 & 44247 & 75.14 \\
\hline 2 & 391.716 & 10363 & 391.725 & 10106 & 79.98 \\
\hline 3 & 392.139 & 4650 & 392.149 & 4488 & 75.81 \\
\hline 4 & 392.727 & 2467 & 392.738 & 2430 & 77.27 \\
\hline 5 & 393.483 & 1640 & 393.495 & 1662 & 82.49 \\
\hline
\end{tabular}

simulations for several axial modes are plotted in Fig. 2. The resulting resonance frequencies and diffractive quality factors are given in Table I and are in excellent agreement with the values obtained in [3]. However, the output mode purity $\left(\mathrm{TE}_{8,5}\right.$ mode content at the cavity output) is rather poor and might, therefore, prohibit the use of such a cavity in an experimental gyrotron.

\section{IMPROVED STEP-TyPE COUPLING}

Circular waveguide modes with the same azimuthal but different radial indices can be effectively coupled by applying a simple abrupt step in the waveguide diameter. To investigate the influence of the radial step in the cavity given in Fig. 1 on the output mode purity, the output radius of a simple stepped waveguide with a fixed input radius $R_{1}=2.588 \mathrm{~mm}$ and $100 \% \mathrm{TE}_{8,4}$ as input mode was varied at a constant frequency of $f=391.471 \mathrm{GHz}$. The length of the straight waveguide sections at both ends of the abrupt step is $10 \mathrm{~mm}$. As wall material, we chose high-purity copper at room temperature with a conductivity of $5.6 \cdot 10^{7} \mathrm{~S} / \mathrm{m}$. The resulting reflected and transmitted modal power as a function of the output radius $R_{2}$ is plotted in Fig. 3. Whereas the reflection occurs in the pure $\mathrm{TE}_{8,4}$ mode, step-type coupling to modes with higher radial indices occurs in forward direction. As can be seen from Fig. 3(c), there is also conversion to many modes with lower radial indices, which is most severe at radii $R_{2}$ where the higher-order modes are close to cutoff. Unfortunately, these are exactly the radii where gyrotron oscillations take place. Fig. 4 shows the calculated reflection and transmission as a
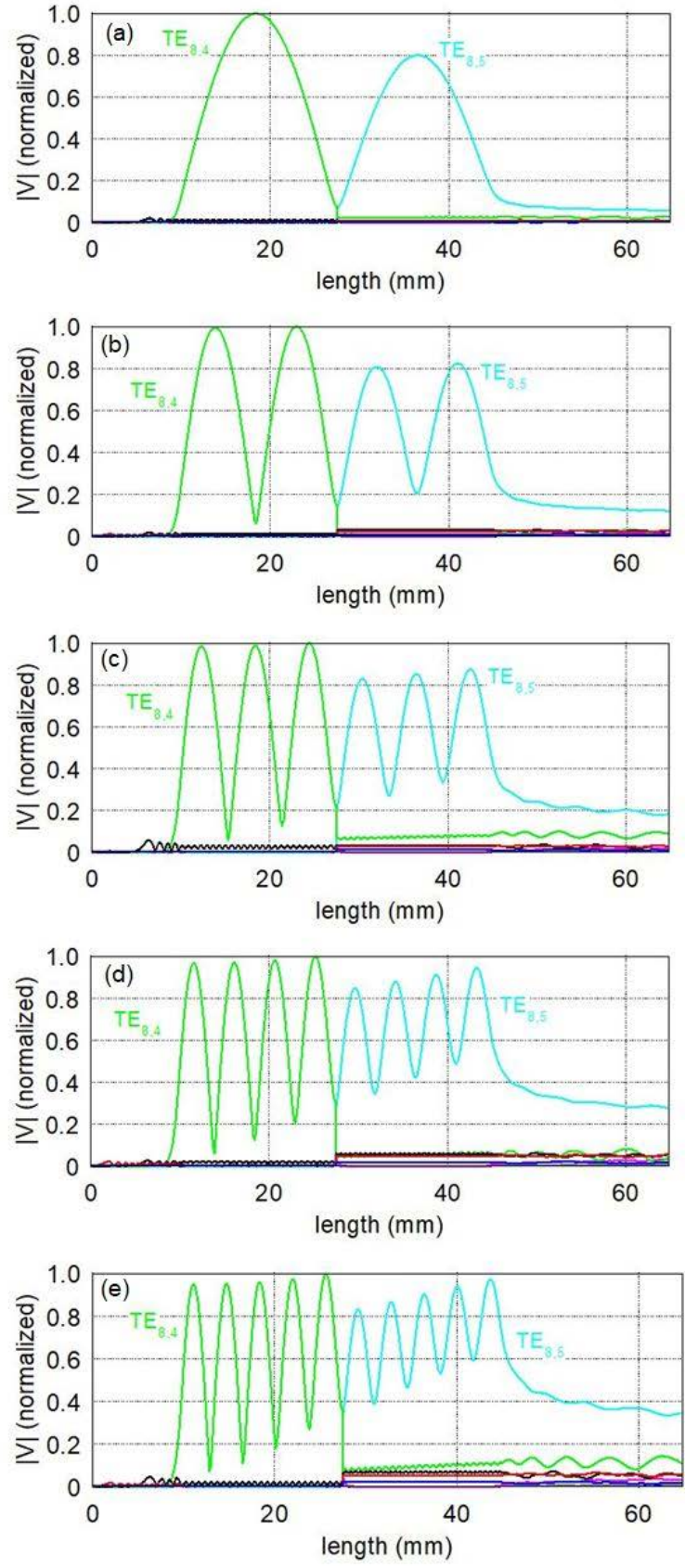

Fig. 2. Calculated axial profiles of the first five antiphase axial modes in the complex $\mathrm{TE}_{8,4, q}-\mathrm{TE}_{8,5, q}$ mode gyrotron cavity from [3]. (a) $q=1$. (b) $q=2$. (c) $q=3$. (d) $q=4$. (e) $q=5$.

function of frequency for the waveguide step of the complex gyrotron cavity plotted in Fig. 1 [3] with $R_{1}=2.588 \mathrm{~mm}$ and $R_{2}=2.9974 \mathrm{~mm}$. As can be clearly seen, strong mode conversion in this complex cavity containing a simple abrupt radial step is practically unavoidable. Introduction of a linear $45^{\circ}$ up-taper between the two sections [2], [7] does not change the situation essentially.

Irises in cavities of sub- $\mathrm{THz}$ gyrotrons have been applied mainly to enhance the diffractive quality factor of the resonators [2], [13]-[15]. Here, we use a radial stub to improve the output mode purity. Into the complex cavity of Fig. 1 [3], we introduce at the end of the first cavity section with radius 

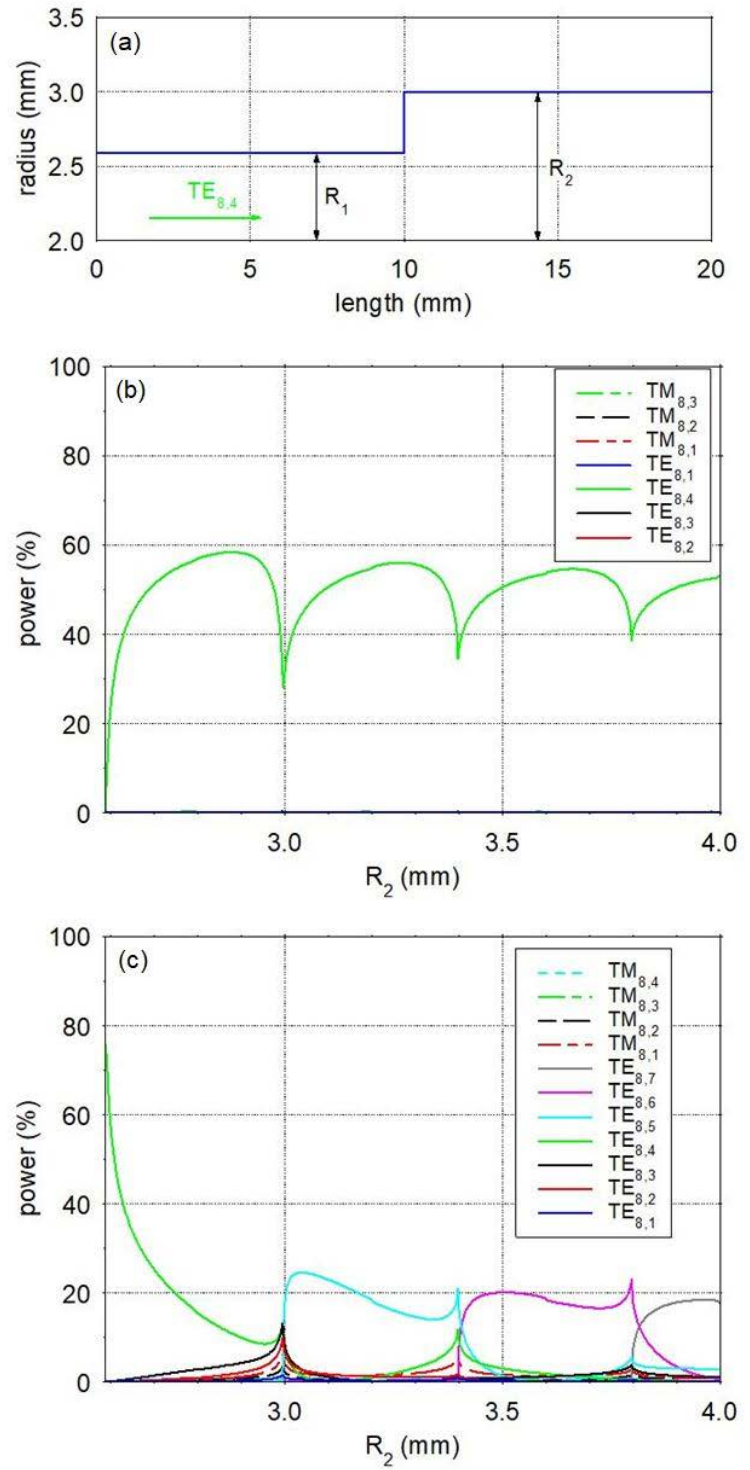

Fig. 3. Abrupt waveguide step with constant input radius $R_{1}=2.588 \mathrm{~mm}$ fed by the $T E_{8,4}$ mode at a frequency of $391.471 \mathrm{GHz}$. (a) Radial contour, (b) calculated reflection, and (c) transmission/mode conversion as a function of the output radius $R_{2}$. In (b), the corresponding dependencies for other modes than $\mathrm{TE}_{8,4}$ are not vis ble due to the selected scale.

$R_{1}$ a larger radial step to an intermediate radius $R_{i}$ followed by a short intermediate section with length $L_{i}$ and then a small radial step down to the radius $R_{2}$ of the second cavity section (Fig. 5). The dimensions of the stub were optimized numerically. Fig. 6 gives the calculated output mode purity of the $\mathrm{TE}_{8,5}$ mode in the larger waveguide section with $R_{2}=$ $2.9974 \mathrm{~mm}$ for $\mathrm{TE}_{8,4}$ as the input mode in the smaller cavity section with $R_{1}=2.588 \mathrm{~mm}$. There is a wide range of parameters $R_{i}$ and $L_{i}$ providing the lowest undesired mode conversion with about $81 \%$ coupling to the $\mathrm{TE}_{8,5}$ mode. We chose the smallest possible dimensions with $R_{i}=3.15 \mathrm{~mm}$ and $L_{i}=0.42 \mathrm{~mm}$, indicated by the black triangle in Fig. 6 . The electric field distributions along the waveguide radial step from $R_{1}=2.588 \mathrm{~mm}$ to $R_{2}=2.9974 \mathrm{~mm}$ without stub and with optimized stub at the frequency of $391.471 \mathrm{GHz}$ are plotted in Fig. 7. Obviously, the function of the stub is
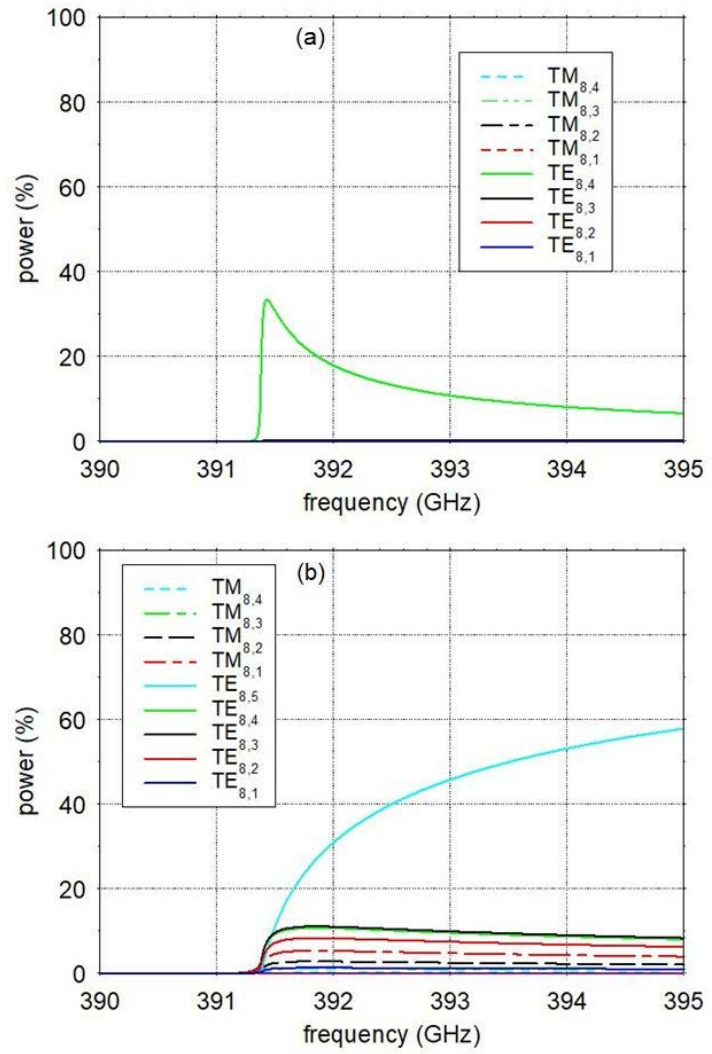

Fig. 4. (a) Calculated reflection and (b) transmission/conversion of the $\mathrm{TE}_{8,4}$ mode at a waveguide step with input radius $R_{1}=2.588 \mathrm{~mm}$ and output radius $R_{2}=2.9974 \mathrm{~mm}$ as a function of frequency. In (a), the corresponding dependencies for other modes than $\mathrm{TE}_{8,4}$ are not visible due to the selected scale.

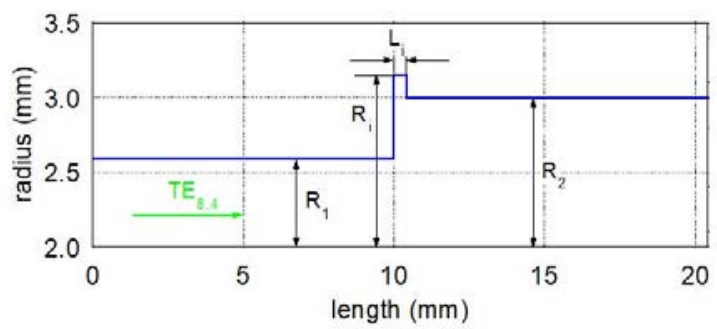

Fig. 5. Radial contour of waveguide step with radial stub and input radius $R_{1}=2.588 \mathrm{~mm}$ and output radius $R_{2}=2.9974 \mathrm{~mm}$.

to reconvert most of the spectrum of the excited propagating lower order and evanescent higher-order modes $\mathrm{TE}_{8, n}$ and $\mathrm{TM}_{8, n}$ into the desired $\mathrm{TE}_{8,5}$ mode. Fig. 8 gives the calculated reflection and transmission of the radial step with optimized stub as a function of frequency. The $\mathrm{TE}_{8,5, q}$-mode contents in the transmitted mode spectrum of the radial step with and without radial stub are compared in Table II for the resonance frequencies of the first five axial modes, showing the substantial improvement of mode purity by the optimized stub.

\section{Complex Cavity With Radial Stub}

We replaced the simple abrupt step in the complex cavity of [3] with the radial stub described in Section III. All other dimensions of the cavity remain the same as in Fig. 1. 


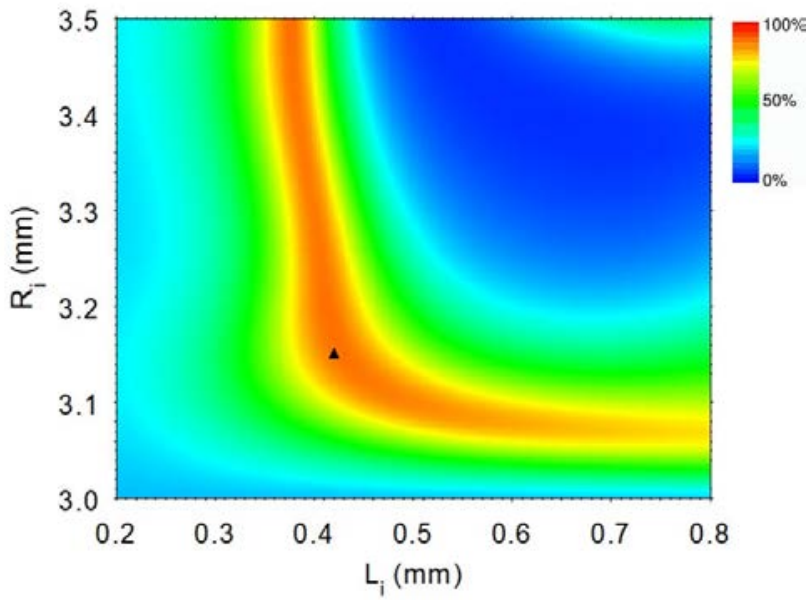

Fig. 6. Calculated output mode purity (percentage of the $\mathrm{TE}_{8,5}$ mode power in the output mode spectrum) of a waveguide step as a function of the radial stub dimensions $\left(L_{i}, R_{i}\right)$ for an input radius of $R_{1}=2.588 \mathrm{~mm}$ and an output radius of $R_{2}=2.9974 \mathrm{~mm}$ at $f=391.471 \mathrm{GHz}$. The smallest possible stub dimensions with optimal coupling are indicated by the black triangle.
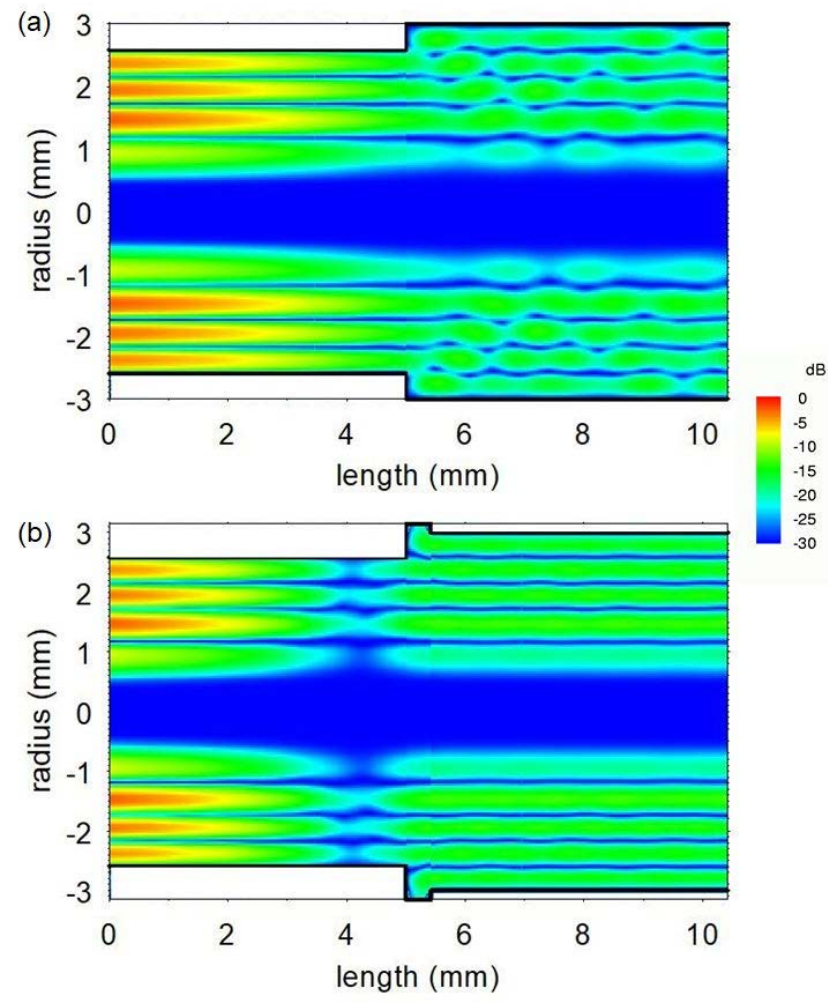

Fig. 7. Calculated azimuthal electric field distribution at a waveguide step from $R_{1}=2.588 \mathrm{~mm}$ to $R_{2}=2.9974 \mathrm{~mm}$ (a) without and (b) with optimized stub with $R_{i}=3.15 \mathrm{~mm}$ and $L_{i}=0.42 \mathrm{~mm}$ with $100 \% \mathrm{TE}_{8,4}$ as input mode at the frequency of $f=391.471 \mathrm{GHz}$.

The geometry of the modified complex cavity with stub is plotted in Fig. 9. Fig. 10 shows the calculated axial field profiles for the first five antiphase axial modes. The comparison with results from the original cavity design in Table III and Fig. 2 shows a significant improvement with respect to the achieved output mode purity. The diffractive quality factor of the complex cavity also increased due to the radial stub, which
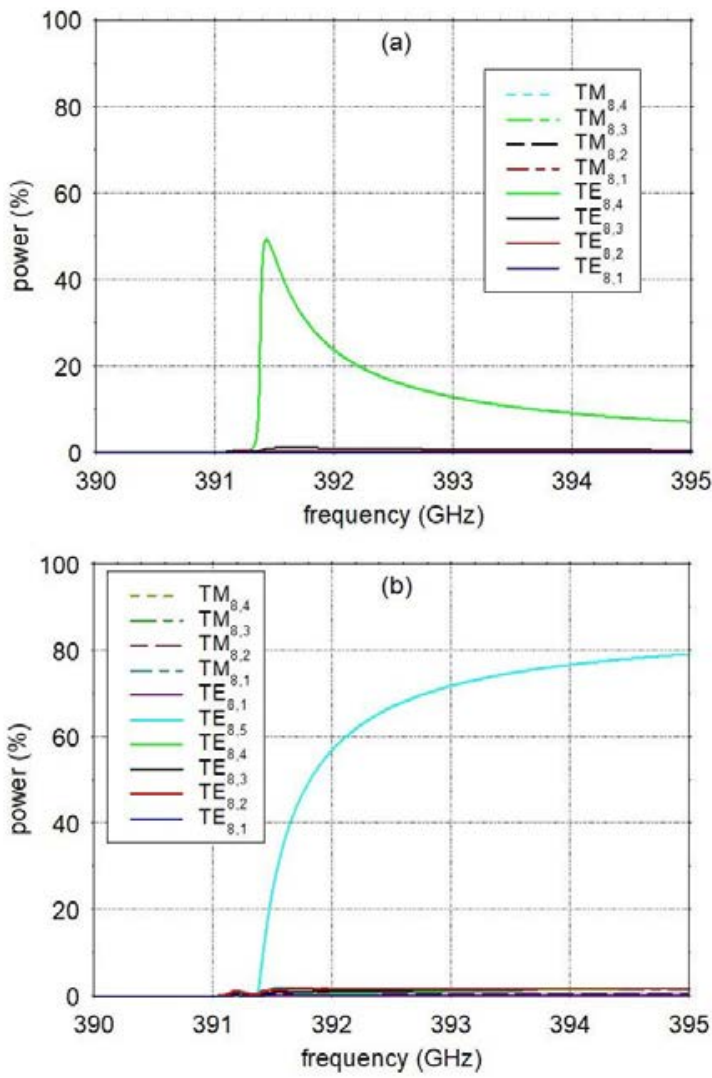

Fig. 8. (a) Calculated reflection and (b) transmission/conversion of the radial step from $R_{1}=2.588 \mathrm{~mm}$ to $R_{2}=2.9974 \mathrm{~mm}$ with optimized stub with $R_{i}=3.15 \mathrm{~mm}$ and $L_{i}=0.42 \mathrm{~mm}$ as a function of frequency.

TABLE II

Comparison of THE MOde ConVERSION OF THE FIRST FIVE ANTIPHASE AXIAL TE $8,5, q$ MODES AT A SIMPLE RADIAL STEP FROM

$R_{1}=2.588 \mathrm{~mm}$ TO $R_{2}=2.9974 \mathrm{~mm}$ WITH A STUB-LOADED STEP WITH $R_{i}=3.15 \mathrm{~mm}$ AND $L_{i}=0.42 \mathrm{~mm}$

\begin{tabular}{|c|l|c|c|}
\hline $\mathrm{q}$ & $f_{\text {res }}(\mathrm{GHz})$ & $\mathrm{TE}_{8,5}(\%)$, simple step & $\mathrm{TE}_{8,5}(\%)$, step with stub \\
\hline 1 & 391.471 & 21.19 & 80.95 \\
\hline 2 & 391.721 & 35.65 & 88.65 \\
\hline 3 & 392.138 & 45.79 & 90.94 \\
\hline 4 & 392.720 & 53.16 & 91.70 \\
\hline 5 & 393.465 & 58.72 & 91.84 \\
\hline
\end{tabular}

is due to both, the slightly increased reflection at the stub compared to the simple abrupt step and the improved mode purity in the cavity.

\section{Optimized Nonlinear Complex CAVITY WITH STUB}

The final output radius of experimental gyrotrons is usually much larger than that given in [3]. In order to determine the output mode purity of a gyrotron, the whole output taper section has to be taken into account. In the case of a simple linear output taper design, which very often is used in experimental devices, this can lead to large fractions of parasitic modes in the output radiation of the gyrotron. To demonstrate this, we assumed a final output window radius 


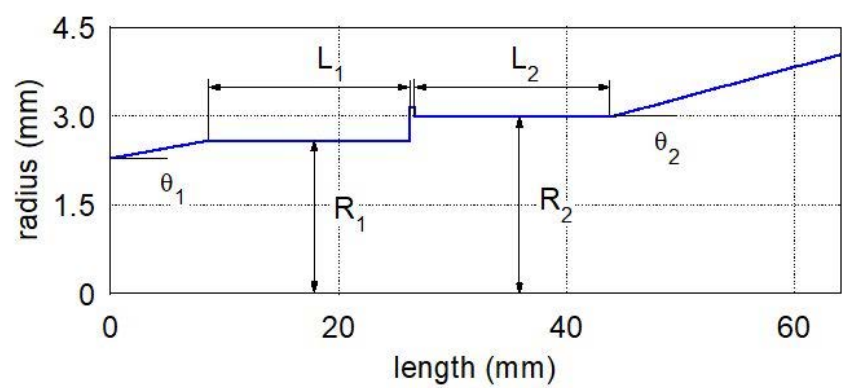

Fig. 9. Radial contour of the complex $\mathrm{TE}_{8,4, q} / \mathrm{TE}_{8,5, q}$ mode gyrotron cavity with intermediate radial stub.

TABLE III

Calculated Resonance Frequencies $f_{\text {res }}$, Diffractive Quality FACTORS $Q_{\text {dif }}$, AND OUTPUT MODE PURITIES OF THE FIRST FIVE ANTIPHASE AXIAL TE T, $_{8, q}$ MODES FOR THE CAVITY WITH RADIAL STUB

\begin{tabular}{|c|c|c|c|}
\hline $\mathrm{q}$ & $f_{\mathrm{res}}(\mathrm{GHz})$ & $Q_{\text {dif }}$ & $\mathrm{TE} 8,5 \%)$ \\
\hline 1 & 391.471 & 57232 & 87.51 \\
\hline 2 & 391.721 & 14868 & 92.55 \\
\hline 3 & 392.138 & 6761 & 89.98 \\
\hline 4 & 392.720 & 3839 & 90.77 \\
\hline 5 & 393.465 & 2597 & 93.92 \\
\hline
\end{tabular}

of $9.0 \mathrm{~mm}$, similar to gyrotron FU VA described in [14]. The output mode purity can be increased significantly with a rounded nonlinear cavity design [13]. A schematic rounding is shown in Fig. 11.

The main parameters are the angles of the adjacent tapers $\theta_{a}$ and $\theta_{b}$ and the length of the rounded section $l$. The radial contour of a rounded section with length $l$, matching the gradients of the adjacent tapers is then given by the following equation:

$$
r(z)=r_{a}+z \cdot\left[\tan \theta_{a}+z \cdot\left(\frac{3 \cdot A-B}{2}+z \cdot \frac{B-A}{2 \cdot l}\right)\right]
$$

with

$$
\begin{aligned}
A & =\frac{r_{b}-r_{a}-\tan \theta_{a} \cdot l}{l^{2}} \\
B & =\frac{\tan \theta_{b}-\tan \theta_{a}}{2 \cdot l} .
\end{aligned}
$$

Based on the original linear complex cavity (Fig. 1), roundings were introduced both at the upstream $\left(l_{1}\right)$ and downstream tapers $\left(l_{2}, l_{3}\right.$, see also the schematic drawing in Fig. 12).

A numerical optimization using the scattering matrix code revealed a maximum output mode purity and a similar diffractive quality factor compared to the linear complex cavity for the following parameters of the nonlinear cavity with radial stub and an output radius of $9.0 \mathrm{~mm}: \theta_{1}=2^{\circ}, \theta_{2}=4^{\circ}, l_{1}=$ $2 \mathrm{~mm}, l_{2}=4 \mathrm{~mm}, l_{3}=95 \mathrm{~mm}$, and $L_{3}=139.5 \mathrm{~mm}$. The contour of the optimized nonlinear cavity with a radial stub is plotted in Fig. 13.
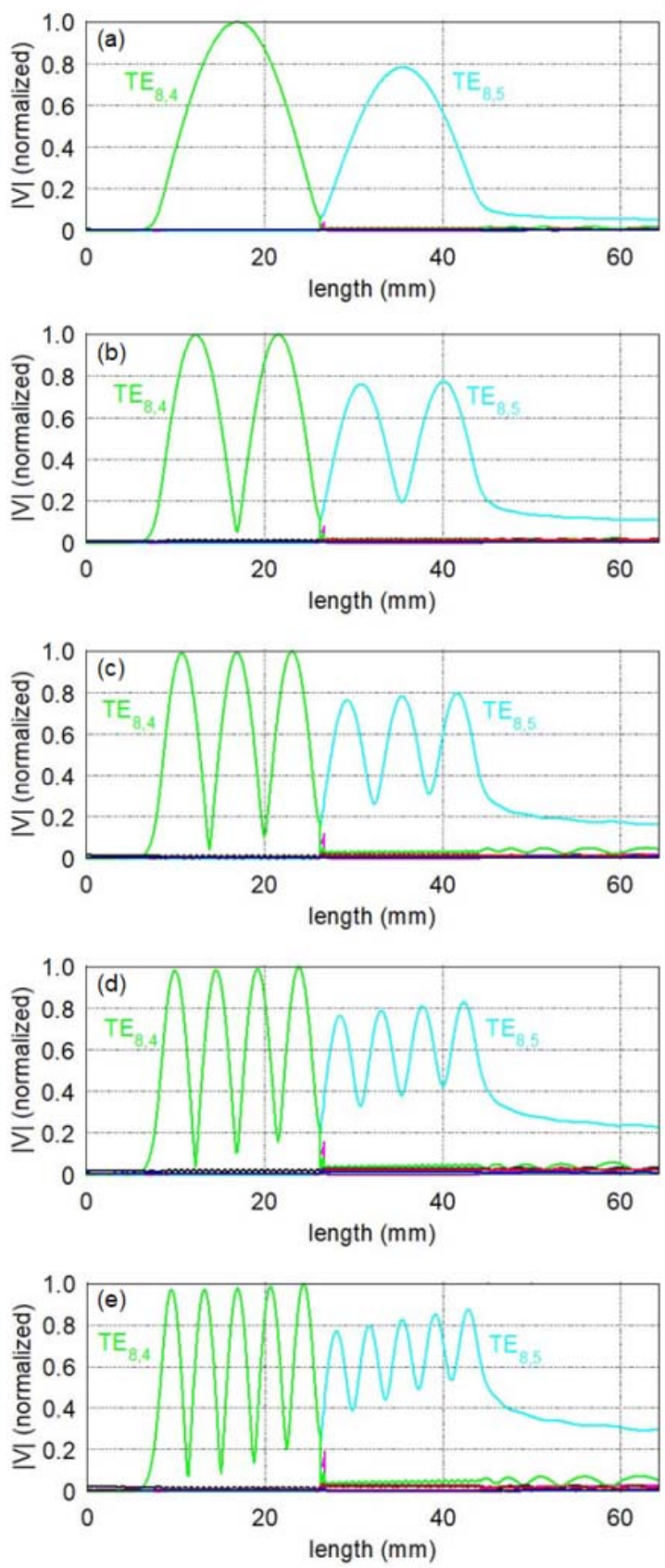

Fig. 10. Calculated axial profiles of the first five antiphase axial modes in the complex $\mathrm{TE}_{8,4, q}-\mathrm{TE}_{8,5, q}$ mode cavity with intermediate stub. (a) $q=$ 1. (b) $q=2$. (c) $q=3$. (d) $q=4$. (e) $q=5$.

Table IV compares the performance of linear input and output tapers with the nonlinear tapers of the optimized cavity design (Fig. 13). It contains the calculated mode purity of the $\mathrm{TE}_{8,4, q}$ modes reflected at the input taper and of the $\mathrm{TE}_{8,5, q}$ mode transmitted through the output taper for $q=1-5$. For both tapers the nonlinear design leads to higher mode purities compared to the linear contour. There is substantial improvement especially for the output taper.

Finally, we compare the output mode purity of the original linear complex cavity of [3] in combination with a linear output up-taper from $R_{2}=2.9974 \mathrm{~mm}$ to $R_{3}=9.0 \mathrm{~mm}$ with 


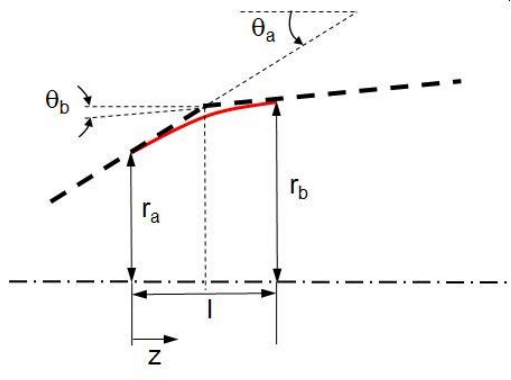

Fig. 11. Rounding between two adjacent circular waveguide tapers.

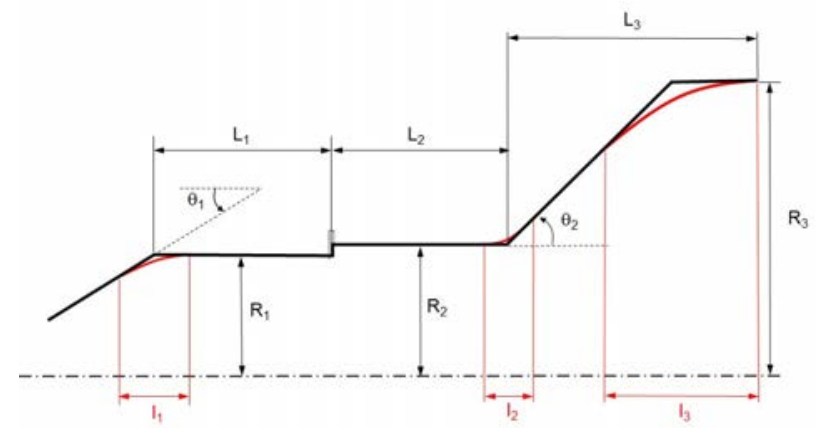

Fig. 12. Geometrical parameters of the nonlinear cavity design with roundings.

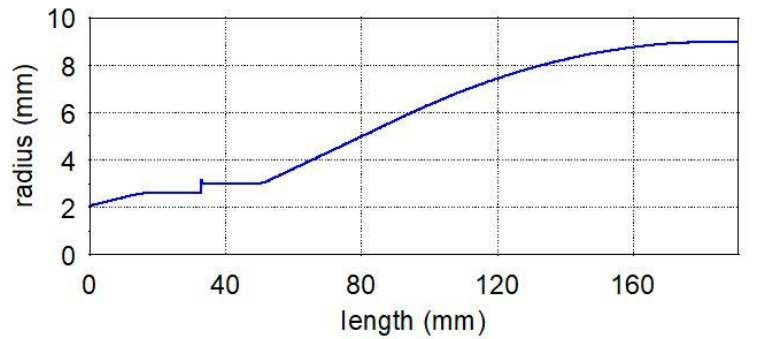

Fig. 13. Radial contour of the optimized nonlinear complex cavity with intermediate stub.

TABLE IV

Comparison of the Calculated Reflection of the TE 8,4 Mode AT THE DOWN-TAPER AND THE TRANSMISSION OF THE TE 8,5 MODE THROUGH THE UP-TAPER BETWEEN THE LINEAR (FIG. 1) AND THE NONLINEAR (FIG. 13) CAVITY DESIGN AT THE RESONANCE FREQUENCIES OF THE COMPLEX CAVITY FOR THE FIRST FIVE ANTIPHASE AXIAL TE $\mathrm{TE}_{8,4, q} / \mathrm{TE}_{8,5, q}$ MODES

\begin{tabular}{|c|c|c|c|c|c|}
\hline & & \multicolumn{2}{|c|}{$\mathrm{TE}_{8,4}(\%)$ down-taper } & \multicolumn{2}{c|}{$\mathrm{TE}_{8,5}(\%)$ up-taper } \\
\hline $\mathrm{q}$ & $f_{\text {res }}(\mathrm{GHz})$ & linear & rounded & linear & rounded \\
\hline 1 & 391.475 & 99.15 & 99.99 & 79.37 & 99.65 \\
\hline 2 & 391.739 & 98.68 & 99.99 & 79.70 & 99.62 \\
\hline 3 & 392.175 & 98.33 & 99.99 & 80.06 & 99.58 \\
\hline 4 & 392.783 & 98.18 & 99.99 & 80.53 & 99.52 \\
\hline 5 & 393.557 & 98.28 & 99.99 & 80.91 & 99.46 \\
\hline
\end{tabular}

the optimized nonlinear design (Fig. 13). Fig. 14 shows the axial field profiles of the first five axial modes in the complete optimized nonlinear geometry. The output mode purities of
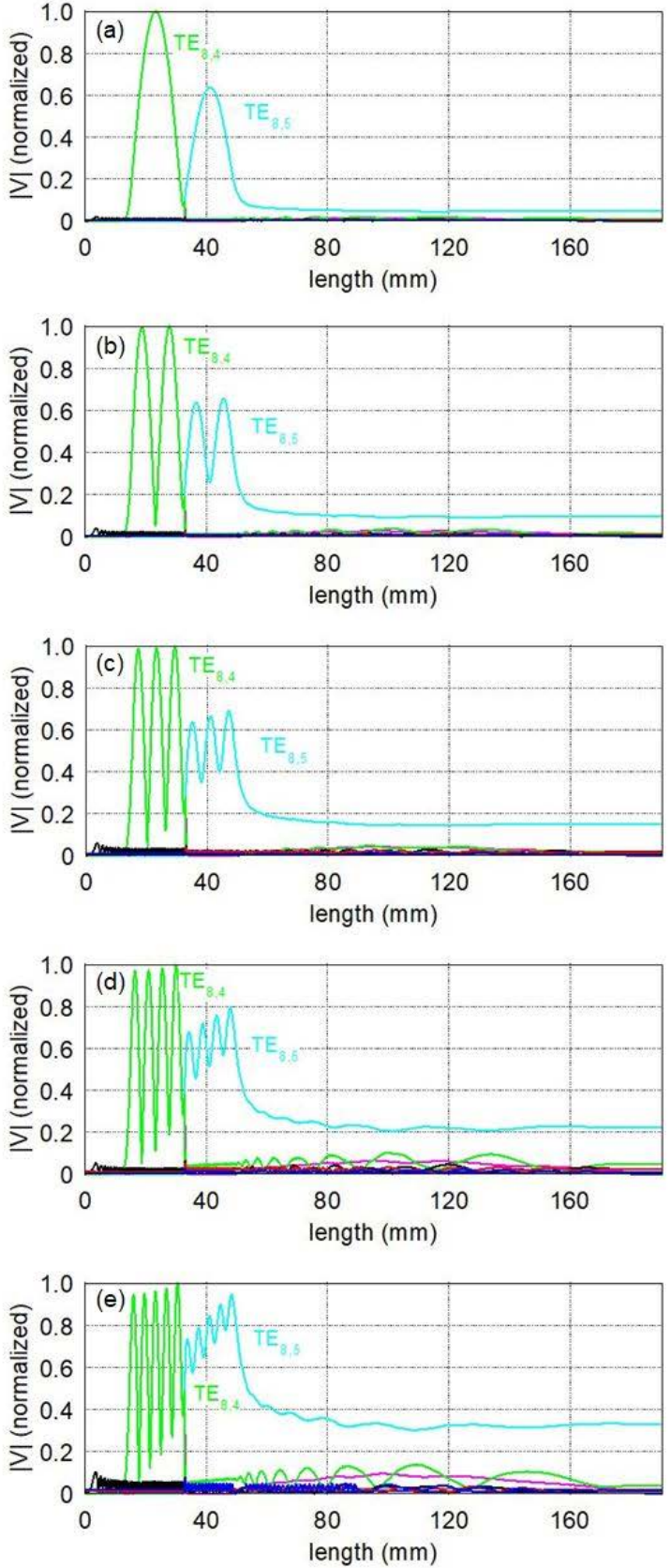

Fig. 14. Calculated axial profiles of the first five antiphase axial modes of the optimized complex cavity with intermediate stub and nonlinear output taper from $R_{2}=2.9974 \mathrm{~mm}$ to $R_{3}=9.0 \mathrm{~mm}$. (a) $q=1$. (b) $q=2$. (c) $q=3$. (d) $q=4$. (e) $q=5$.

the linear and the nonlinear gyrotron designs at the resonance frequencies are compared in Table V.

The very poor output mode purity of the linear gyrotron design results from both-mode conversion at the step in the cavity and in the linear output taper. With the addition of the intermediate radial stub in the complex cavity in combination with an optimized nonlinear cavity profile the overall mode conversion could be successfully reduced to just a few percent for all the considered five axial cavity modes. 
TABLE V

Comparison of the Calculated Resonance Frequencies, and OUtPut MOdE PURITIES OF THE COMPLEX CAVITY OF [3] WITH LINEAR TAPER FROM RADIUS $R_{2}=2.9974 \mathrm{~mm}$ TO $R_{3}=9.0 \mathrm{~mm}$ AND Constant TAPER ANGLE OF $\theta_{2}=3^{\circ} \mathrm{W}$ ITH THE COMPLEX CAVITY DESIGN With StUB AND NONLINEAR TAPERS FOR the FIRST FIVE ANTIPHASE AXIAL TE $\mathrm{T}_{8,4, q} / \mathrm{TE}_{8,5, q}$ MODES

\begin{tabular}{|c|c|c|c|c|c|c|}
\hline & \multicolumn{3}{|c|}{$\begin{array}{c}\text { Cavity with simple step and linear } \\
\text { input and output tapers }\end{array}$} & \multicolumn{3}{c|}{$\begin{array}{c}\text { Cavity with stub and nonlinear } \\
\text { input and output tapers }\end{array}$} \\
\hline $\mathrm{q}$ & fres $_{\text {res }}(\mathrm{GHz})$ & $Q_{\text {dif }}$ & $\mathrm{TE}_{8,5}(\%)$ & $f_{\text {res }}(\mathrm{GHz})$ & $Q_{\text {dif }}$ & $\mathrm{TE}_{8,5}(\%)$ \\
\hline 1 & 391.472 & 44097 & 67.71 & 391.475 & 47974 & 96.36 \\
\hline 2 & 391.725 & 10141 & 67.41 & 391.739 & 12349 & 95.54 \\
\hline 3 & 392.149 & 4533 & 61.68 & 392.175 & 5614 & 95.01 \\
\hline 4 & 392.738 & 2493 & 74.01 & 392.783 & 3037 & 92.48 \\
\hline 5 & 393.495 & 1733 & 55.09 & 393.557 & 1921 & 96.66 \\
\hline
\end{tabular}

\section{CONCLUSION}

A complex $\mathrm{TE}_{8,4, q} / \mathrm{TE}_{8,5, q}$-mode cavity for a frequencytunable sub- $\mathrm{THz}$ gyrotron has been analyzed using a multimode resonator code based on the scattering matrix formalism. It has been shown that a simple radial step between the two cavity sections causes significant conversion to parasitic modes, even when the ratio of the radii of the two sections is precisely correct. The introduction of an additional groove (stub) between the two cavity sections removes this mode conversion almost completely for all the five axial modes at the different frequencies from 391.5 to $393.5 \mathrm{GHz}$. Roundings were introduced both at the upstream and downstream tapers of the stepped cavity. By additionally replacing the linear up-taper to collector and output window with an optimized nonlinear taper, in all cases a gyrotron output mode purity of $>92.5 \%$ could be demonstrated. Since the rounding at the cavity output and the output taper angle have been chosen in such a way that the quality factors of the original and the improved stepped cavity are approximately the same, we expect similar electronic efficiencies of the two versions. However, this has to be proved with electron-beam-wave interaction calculations in a following article.

\section{REFERENCES}

[1] Q. Zhao, S. Yu, X. Li, and T. Zhang, "Theoretical study on mode competition between fundamental and second harmonic modes in a 0.42 THz gyrotron with gradually tapered complex cavity," Phys. Plasmas, vol. 22, no. 10, Oct. 2015, Art. no. 103114.

[2] A. Q. Zhao and B. S. Yu, "The nonlinear designs and experiments on a $0.42-\mathrm{THz}$ second harmonic gyrotron with complex cavity," IEEE Trans. Electron Devices, vol. 64, no. 2, pp. 564-570, Feb. 2017, doi: 10.1109/TED.2016.2642984.

[3] M. M. Melnikova, A. G. Rozhnev, and N. M. Ryskin, "Electromagnetic modeling of a complex-cavity resonator for the $0.4-\mathrm{THz}$ second-harmonic frequency-tunable gyrotron," IEEE Trans. Electron Devices, vol. 64, no. 12, pp. 5141-5148, Dec. 2017, doi: 10.1109/TED.2017.2764874.

[4] A. V. Gaponov et al., "Powerful millimetre-wave gyrotrons," Int. J. Electron., vol. 51, no. 4, pp. 277-302, Jul. 1981.

[5] S. A. Malygin, V. G. Pavel'Yev, and E. S. Tsimring, "Resonant transformation of modes in oversize electrodynamic systems," Radiophys. Quantum Electron., vol. 26, no. 9, pp. 1126-1133, Sep. 1983.

[6] Y. Carmel et al., "Realization of a stable and highly efficient gyrotron for controlled fusion research," Phys. Rev. Lett., vol. 50, no. 2, pp. 112-116, 1983.

[7] V. E. Zapevalov, S. A. Malygin, V. G. Pavel'Ev, and S. E. Tsimring, "Coupled-resonator gyrotrons with mode conversion," Radiophys. Quantum Electron., vol. 27, no. 9, pp. 846-852, 1984.

[8] K. Felch et al., "CW operation of a $140 \mathrm{GHz}$ gyrotron," Int. J. Electron., vol. 61, no. 6, pp. 701-714, 1986.

[9] G. V. Pavel'Ev, S. E. Tsimring, and V. E. Zapevalov, "Coupled cavities with mode conversion in gyrotrons," Int. J. Electron., vol. 63, no. 3, pp. 379-391, Feb. 1987.

[10] A. W. Fliflet, R. C. Lee, and M. E. Read, "Self-consistent field model for the complex cavity gyrotron," Int. J. Electron., vol. 65, no. 3, pp. 273-283, Nov. 1987.

[11] K. Felch, H. Huey, and H. Jory, "Gyrotrons for ECH applications," J. Fusion Energy, vol. 9, no. 1, pp. 59-75, 1990.

[12] Y. Huang, H. Li, S. Yang, and S. Liu, "Study of a 35-GHz third-harmonic low-voltage complex cavity gyrotron," IEEE Trans. Plasma Sci., vol. 27, no. 2, pp. 368-373, Apr. 1999

[13] D. Wagner, G. Gantenbein, W. Kasparek, and M. Thumm, "Improved gyrotron cavity with high quality factor," Int. J. Infr. Millim. Waves, vol. 16, no. 9, pp. 1481-1489, Sep. 1995, doi: 10.1007/ BF02274811.

[14] T. Idehara et al., "Observation of mode patterns for high purity mode operation in the submillimeter wave gyrotron FU VA," Int. J. Infr. Millim. Waves, vol. 23, no. 7, pp.973-980, 2002, doi: 10.1023/A:1019666800167.

[15] A. S. Zuev, A. S. Sedov, E. S. Semenov, A. P. Fokin, and M. Y. Glyavin, "Analysis of the possibilities to control diffraction quality factors of the cavities of subterahertz gyrotrons," IEEE Trans. Plasma Sci. vol. 48, no. 11, pp. 4037-4040, Nov. 2020, doi: 10.1109/TPS.2020. 3025689 . 
Karlsruher Institut für Technologie

\section{Repository KITopen}

Dies ist ein Postprint/begutachtetes Manuskript.

Empfohlene Zitierung:

Wagner, D.; Thumm, M.

Improvement of the Output Mode Purity of a Complex-Cavity Resonator for a Frequency-

Tunable Sub-THz Gyrotron.

2021. IEEE Transactions on Electron Devices, 68

doi: $10.5445 / / R / 1000137479$

Zitierung der Originalveröffentlichung:

Wagner, D.; Thumm, M.

Improvement of the Output Mode Purity of a Complex-Cavity Resonator for a FrequencyTunable Sub-THz Gyrotron.

2021. IEEE Transactions on Electron Devices, 68 (10), 5220-5226.

doi:10.1109/TED.2021.3105955 